\title{
Building networks with MIMO and OFDM Technology
}

\author{
Chengwei $\mathrm{Hu}$ \\ Electronic Communication Department, Guanghou Civil Aviation College, Guangzhou, China \\ huchengwei@caac.net
}

\begin{abstract}
A multiple input multiple output (MIMO) system provides multiple independent transmission channels, thus, under certain conditions, leading to a channel capacity that increases linearly with the number of antennas. Orthogonal frequency division multiplexing (OFDM) is known as an effective technique for high data rate wireless mobile communication. By combining these two promising techniques, the MIMO and OFDM techniques, we can significantly increase data rate, which is justified by improving bit error rate (BER) performance., it is an attractive air-interface solution for next-generation wireless local area networks (WLANs), wireless metropolitan area networks (WMANs), and fourth-generation mobile cellular wireless systems. This article also provides an overview of the basics of MIMO-OFDM technology and focuses on space-frequency signaling and multiuser systems. Efficient burst frequency synchronization for a system combining MIMO with OFDM was proposed and analyzed.
\end{abstract}

Key words: MIMO-OFDM; Multiple-input; Multiple-output; Synchronization; Building networks

\section{INTRODUCTION}

Orthogonal frequency division multiplexing (OFDM) has become a popular technique for transmission of signals over wireless channels. OFDM has been adopted in several wireless standards such as digital audio broadcasting (DAB), digital video broadcasting (DVB-T), the IEEE 802.11a [1] local area network (LAN) standard and the IEEE 802.16a [2] metropolitan area network (MAN) standard. OFDM converts a frequency selective channel into a parallel collection of frequency flat sub-channels. The subcarriers have the minimum frequency separation required to maintain orthogonality of their corresponding time domain waveforms, yet the signal spectra corresponding to the different subcarriers overlap in frequency. Hence, the available bandwidth is used very efficiently. If knowledge of the channel is available at the transmitter, then the OFDM transmitter can adapt its signaling strategy to match the channel. Since OFDM uses a large collection of narrowly spaced subchannels, these adaptive strategies can approach the ideal water pouring capacity of a frequency selective channel. In practice this is achieved by using adaptive bit loading techniques, where different sized signal constellations are transmitted on the subcarriers.

Multiple antennas can be used at the transmitter and receiver, an arrangement called a multiple-input multipleoutput (MIMO) system. A MIMO system takes advantage of the spatial diversity that is obtained by spatially separated antennas in a dense multipath scattering environment. MIMO systems may be implemented in several different ways to obtain either a diversity gain to combat signal fading, or to obtain a capacity gain. Generally, there are three categories of MIMO techniques. The first aims to improve the power efficiency by maximizing spatial diversity. Such techniques include delay diversity, space-time block codes (STBC) [4], [5] and spacetime trellis codes (STTC) [6]. The second class uses a layered approach to increase capacity. One popular example of such a system is V-BLAST suggested by Foschini et al. [7] where full spatial diversity is usually not achieved. Finally, the third type exploits the knowledge of channel at the transmitter. It decomposes the channel coefficient matrix using Singular Value Decomposition (SVD) and uses these decomposed unitary matrices as pre and post filters at the transmitter and the receiver to achieve near capacity [8].

The idea of using multiple receive and multiple transmit antennas has emerged as one of the most significant technical breakthroughs in modern wireless communications. Theoretical studies and initial prototyping of these 
MIMO systems have shown order of magnitude spectral efficiency improvements in communications. As a result, MIMO is considered a key technology for improving the throughput of future wireless broadband data systems.

High-performance 4th generation (4G) broadband wireless communication enabled by the use of multiple antennas not only at transmitter multiple input multiple output (MIMO) system provides multiple channels, thus, under certain conditions, leading to a channel capacity with the number of antennas. Orthogonal frequency division known as an effective technique for high data rate wireless combining these two promising techniques, the MIMO and OFDM significantly increase data rate, which is justified by improving performance. In this section, we briefly describe the concept of comparison with CDMA system; its key benefits are discussed.

\section{MIMO-OFDM SYSTEM MODEL}

The specifications of the MIMO-OFDM system under consideration are derived from the IEEE 802.11a WLAN standard; the corresponding high-level block diagram is shown in Figure 1. In the transmitter, the high-rate data stream is first split into four parallel lower rate streams, which are OFDM modulated (according to the IEEE 802.11a physical layer parameters summarized in Table 1) and sent through the MIMO channel. The received signals, consisting of a superposition of all transmitted signals, are then OFDM demodulated, spatially separated and demultiplexed into a single high-rate stream. Our design does not include forward error correction coding.

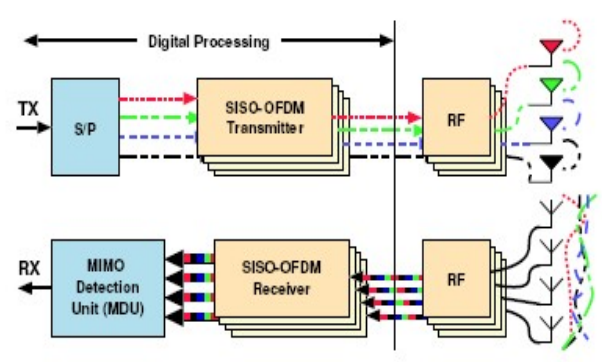

FIGURE 1: High-level block diagram of a MIMO-OFDM transceiver (top: transmitter, bottom: receiver).

Traditionally, multiple antennas (at one side of the wireless link) have been used to perform interference cancellation and to realize diversity and array gain through coherent combining. The use of multiple antennas at both sides of the link offers an additional fundamental gain — spatial multiplexing gain, which results in increased spectral efficiency. A brief review of the techniques in a MIMO system is given in the following.

Spatial multiplexing yields a linear (in the minimum of the number of transmit and receive antennas) capacity increase, compared to systems, with a single antenna at one or both side of the link, at no additional power or bandwidth expenditure. The corresponding gain is available if the propagation channel exhibits rich scattering and can be realized by the simultaneous transmission of independent data streams in the same frequency band. The receiver exploits differences in the spatial signatures induced by the MIMO channel onto the multiplexed data streams to separate the different signals, thereby realizing a capacity gain.

Diversity leads to improved link reliability by rendering the channel "less fading" and by increasing the robustness to co-channel interference. Diversity gain is obtained by transmitting the data signal over multiple (ideally) independently fading dimensions in time, frequency, and space and by performing proper combining in the receiver. Spatial (i.e., antenna) diversity is particularly attractive when compared to time or frequency diversity, as it does not incur expenditure in transmission time or bandwidth, respectively. Space-time coding realizes spatial diversity gain in systems with multiple transmit antennas without requiring channel knowledge at the transmitter. Array gain can be realized both at the transmitter and the receiver. It requires channel knowledge for coherent combining and results in an increase in average receive signal-to-noise ratio (SNR) and hence improved coverage.

\section{MIMO-OFDM SIGNAL MODEL}

Suppose that a communication system consists of $\mathrm{Nt}$ transmit (TX) and $\mathrm{Nr}$ receive (RX) antennas, denoted as a $\mathrm{Nt} \times \mathrm{Nr}$ system. Figure 1 depicts such a system. Let us define the a-th MIMO OFDM vector to be transmitted as ^s(a) $=\operatorname{vec}(\mathrm{s}(0, \mathrm{a}), \mathrm{s}(1, \mathrm{a}), \ldots, \mathrm{s}(\mathrm{Nc}-1, \mathrm{a}))$, where $\mathrm{s}(\mathrm{n}, \mathrm{a})$ denotes the $\mathrm{Nt} \times 1$ frequency domain MIMO transmit vector 
for the n-th subcarrier and Nc represents the number of subcarriers. This vector is transformed to the time domain using the inverse discrete Fourier transform (IDFT)

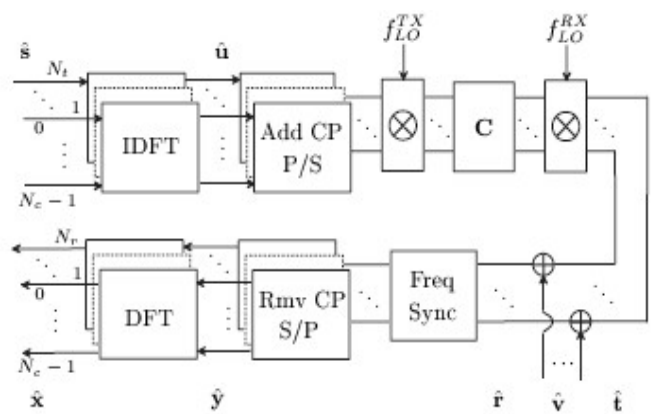

FIGURE 2: MIMO-OFDM system frequency synchronization.

$$
\hat{\mathbf{u}}(a)=\left(\mathbf{F}^{-1} \otimes \mathbf{I}_{N_{t}}\right) \hat{\mathbf{s}}(a)
$$

where $\otimes$ denotes the Kronecker Product, $\mathbf{F}$ is the $N c \times N c$ Fourier matrix, of which the $(i, k)$-th element equals e $\times \mathrm{p}\left(-j 2 \pi \frac{i k}{N}\right)$ and $\mathrm{I} N t$ represents the $N \times N$ dimensional identity matrix. A cyclic prefix (CP) is added to the signal $\widehat{\mathrm{u}}$ (a) by multiplication with matrix $\boldsymbol{\Theta}$, which adds the last $N t N g$ elements of $\widehat{\mathrm{u}}$ (a) on top of $\widehat{\mathrm{u}}(\mathrm{a})$. We assume here that the CP is longer than the channel impulse response (CIR), avoiding inter-symbol-interference (ISI). It is assumed that the average total TX power $P$ is divided among the TX antennas such that $\sigma^{2}=P / N t$. The signal is then converted to radio frequency centered at $f T X / L O$ and transmitted through the quasi-static multipath channel $\mathbf{C}$. The average channel or propagation attenuation is assumed to $b e \sigma^{2}=1$. At the RX the signal is down converted to baseband with the local oscillator centered at $f R X / L O$, where the $a$-th received $N c N r \times 1$ time domain symbol is given by $\hat{\mathrm{r}}(\mathrm{a})=\operatorname{vec}(\mathbf{r}(a \cdot(N c+N g)), \ldots, \mathbf{r}((a+1) \cdot(N c+N g)-1))$, where $\mathbf{r}(m)$ denotes the $N r \times 1$ receive vector at sample instant $m$. The $\mathrm{CP}$ is removed (Rmv CP) by multiplication with $\boldsymbol{\Theta}^{-1}$, which removes the first $N r N g$ elements of $\hat{\mathrm{r}}(\mathrm{a})$. The received signal $\hat{\mathrm{y}}$ is converted to the frequency domain using the DFT, when the CFO is zero, thus $\Delta f=f_{L O}^{T X}-f_{L O}^{R X}=0$, this yields

$$
\begin{aligned}
\hat{x}(a) & =(\mathrm{F} \otimes \mathrm{I} N r) \hat{y}(a)=(\mathrm{F} \otimes \mathrm{I} N r) \theta^{-1} \hat{V}(a) \\
& =(\mathrm{F} \otimes \mathrm{I} N r) \theta^{-1}\left(C \theta\left(\left(\mathrm{F}^{-1} \otimes \mathrm{I} N t\right)\right) \hat{S}(a)+\hat{V}(a)\right) \\
& =H \hat{S}(a)+\hat{u}(a)
\end{aligned}
$$

where $\hat{C}=\mathbf{\Theta}-1 \mathbf{C} \Theta$ is a block circulant matrix signifying the time domain MIMO channel, which can be diagonalized by the IDFT and DFT operation [14] yielding the $N c N r \times N c N t$ block diagonal matrix $\hat{\mathrm{H}}$. The $n$-th $N r$ $\times N t$ block diagonal element is $\mathbf{H}(n)$, the $N r \times N t$ MIMO channel of the $n$-th subcarrier. $\hat{n}(a)$ represents the frequency-domain noise, with i.i.d. zero-mean, complex Gaussian elements and $\hat{V}(a)$ denotes its time-domain equivalent. The variance of the elements of $\mathbf{v}(m)$ is $\sigma^{2} v$. It is clear from (2) that the carriers are orthogonal. When frequency offset occurs, thus $\Delta f \neq 0$, the received frequency domain signal is given by

$$
\begin{aligned}
\hat{x}(a) & =(\mathrm{F} \otimes \mathrm{I} N r) \theta^{-1}\left(\mathrm{EC} \theta\left(\mathrm{F}^{-1} \otimes \mathrm{I} N t\right) \hat{S}(a)+\hat{V}(a)\right) \\
& =(\mathrm{G} \otimes \mathrm{I} N r) \hat{\mathrm{H}} \hat{S}
\end{aligned}
$$


Where $\mathbf{E}=\operatorname{diag}(e 0, e 1, \ldots, e N c+N g-1) \otimes \mathbf{I} N r$ denotes the phase rotation due to the CFO, with $e m=\exp$ $(j 2 \pi \Delta f T s(a(N c+N g)+m))$. Here we assumed that the phase offset at the start of the packet is part of the channel. Ts denotes the sample time. From (3) it is clear that $\mathbf{\Theta}-1 \mathbf{E C \Theta}$ is no longer block circulant and can thus not be diagonalized by the DFT and IDFT operations. The $N c \times N c$ matrix $\mathbf{G}$ in (3) shows the influence of the CFO on the received frequency domain symbols and is given by

$$
G=\left(\begin{array}{ccc}
g_{0} & g_{-1} \ldots & g_{-\left(N_{C-1}\right)} \\
g_{1} & g_{0} & g_{-\left(N_{C-2)}\right.} \\
\vdots & \cdots & \vdots \\
g_{-\left(N_{C-1)}\right.} & g_{-\left(N_{C-2)}\right.} & \cdots g_{0}
\end{array}\right)
$$

Where

$$
\begin{aligned}
\mathrm{g}_{\mathrm{q}}= & \frac{\sin (\pi(\sigma-q))}{N_{c} \sin \frac{\pi}{N_{c}}(\sigma-q)} \exp \left(j \frac{\pi\left(\left(N_{c}-1\right)\right.}{N_{c}}(\sigma-q)\right) \\
& \exp \left(j \frac{2 \pi \sigma}{N_{c}}\left(a\left(N_{c}+N g\right)+N_{g}\right)\right) \sigma
\end{aligned}
$$

where $\sigma=\Delta f N c / f s$ is the frequency offset normalized to the subcarrier spacing. We clearly see the following effects of frequency offset: the wanted carriers, multiplied with $g 0$, are rotated and their amplitude is reduced, and the other elements of $\mathbf{G}$, thus for $q_{-}=0$, introduce cross terms which result in。

\section{MIMO-OFDM FRAME STRUCTURE}

The frame structure proposed in the IEEE 802.11a standard has been adapted to the MIMO case as illustrated in Figure 3. The main difference to the single-antenna case lies in the short preamble sequence and the part of the frame containing the training symbols. The four different short preambles $\operatorname{SPt}(\mathrm{t}=1, \ldots, 4)$ are derived from the original IEEE 802.11a short preamble SP1 by tone-wise multiplication of the non zero entries of SP1 by different Hadamard codes; this avoids unintended beam forming, while still enabling the use of well-known SISO frequency offset estimation algorithms [7]. Compared to SISO systems, more training is needed to estimate the MIMO channel, which is due to the higher number of channel parameters in the MIMO case. To this end, each antenna transmits four OFDM training symbols $\operatorname{Ttn}(\mathrm{t}, \mathrm{n}=1, \ldots, 4)$ instead of the long preamble as specified in the IEEE 802.11a standard. The training symbols are orthogonal across antennas which are achieved by having only one antenna transmit on any given tone. K OFDM data symbols per antenna denoted as Dtk conclude the frame; the individual antennas transmit spatially independent data streams concurrently on all tones. Thus, the system operates in spatial multiplexing mode.

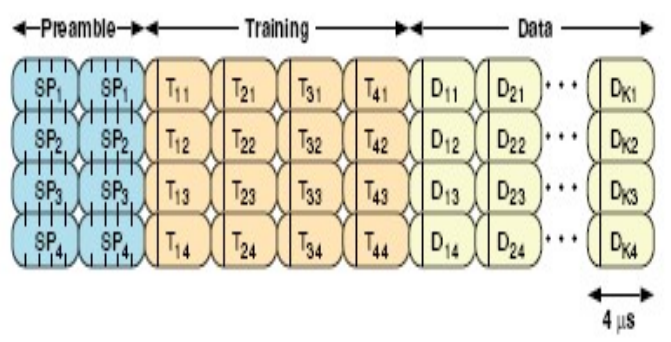

FIGURE 3: MIMO-OFDM frame structure. Each block corresponds to one OFDM symbol. 


\section{PERFORMANCE ANALYSIS}

A MIMO-OFDM architecture that significantly increases the achievable bit rate of the system as well as decreases the Co Channel Interference has been studied and analyzed. Simulation results show the effectiveness of the considered system. For ideal conditions $(?=0)$ and same number of transmit and receive antennas, Figure 4 shows that V-BLAST(Vertical Bell laboratory Layered Space Time) technique for CCI cancellation increases the bit rate with number of antennas (ideally by $\mathrm{N}=\mathrm{M}$ ) without significantly worsening BER. The diversity level on $\mathrm{ZF}$ (zero forcing) V-BLAST is $(\mathrm{M}-\mathrm{N}+1)$ when detecting the first layer. With each layer detected, the diversity level of the resulting system should increase layer by layer, until $\mathrm{N}$ for the last layer, since the detected layers have been cancelled while the receive antennas still keeps constant. However, the diversity level of $(\mathrm{M}-\mathrm{N}+1)$ for the first layer is too low in most cases, which largely limits the error performance of ZF VBLAST. For example, if $N=M$, there would be no diversity gain for the first layer.

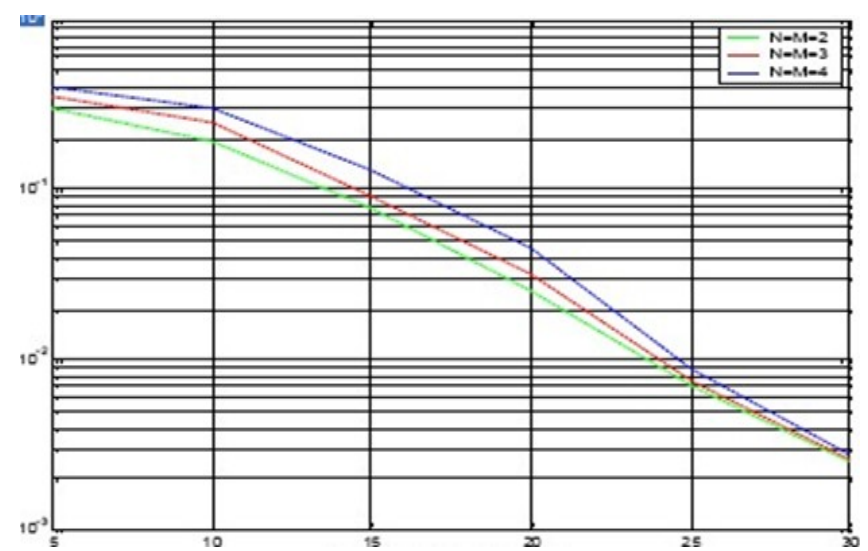

FIGURE 4: System performance comparison for different values of $\mathrm{N}=\mathrm{M}$.

MIMO systems with arrays of different number of receive antennas $(\mathrm{M}=\mathrm{N}$ is required for V-BLAST) are showed in Figure 5. As can be seen, increasing $\mathrm{M}$ improves performance by order of M-N. This is because a greater number of received signals can be combined (diversity) in a more efficient way to obtain a more accurate estimate of the transmitted signals.

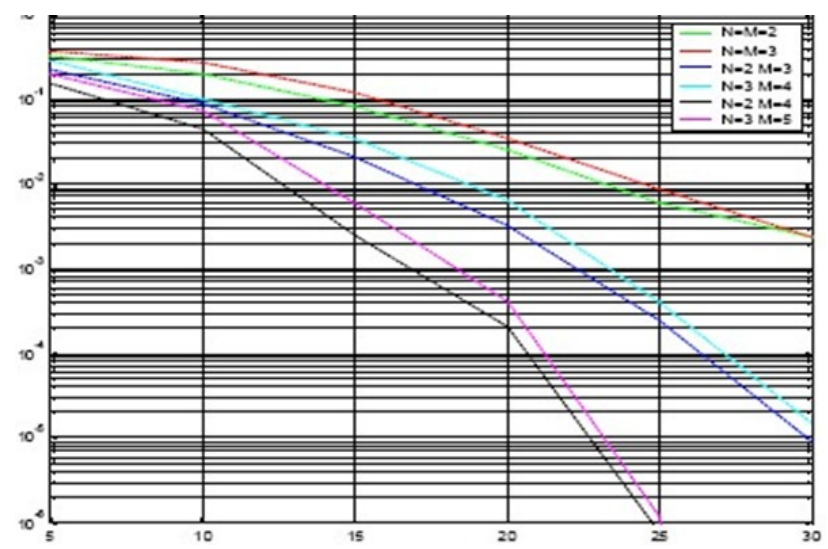

FIGURE 5: System performance comparison for different values of $M-N$

Several studies shown that if channel path gains of a (N,M) MIMO system are completely uncorrelated -even if channel is unknown for the transmitter-, the channel capacity scales linearly with $\mathrm{p}$, where $\mathrm{p}=\min \{\mathrm{N}, \mathrm{M}\}$. However, in practical propagation conditions, these channel coefficients could be partially correlated. Generally the correlation coefficient depend on many factors like the physical parameters of the transmit and receive antennas, i.e. antenna 
spacing, but also on the characteristics and the distribution of the scatterers. Figure 5 shows that when fades are correlated, the channel capacity can be significantly smaller than when they are not, and Figures 6 suggests that the negative effect is more significant for higher modulation schemes.

One solution to improving nulling performance is to replace the ZF Nulling by the more powerful Minimum Mean Square Error (MMSE) Nulling, which improves the system performance over ZF V-BLAST by $6 \mathrm{~dB}$.

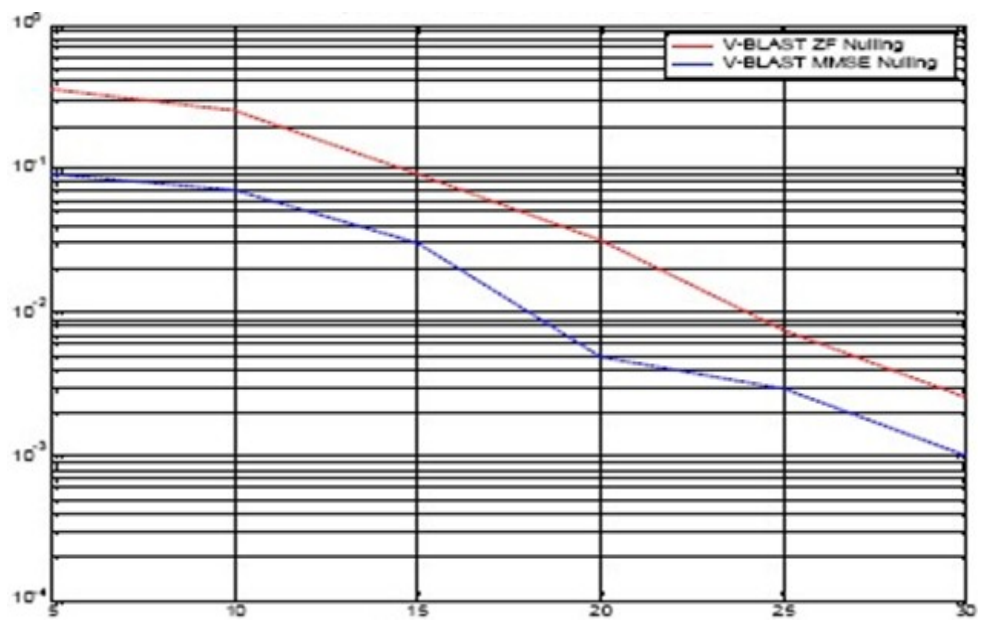

FIGURE 6: Performance of the 3x3 MIMO systems for ZF and MMSE nulling

V-BLAST algorithm based in the QR factorization of $\mathrm{H}$ with $\mathrm{w}(\mathrm{k})=\mathrm{R}$, which can reduce the detection and CCI cancellation complexity in the receiver as presented in [9] and [10], was also tested with similar results to the "traditional" ZF V-BLAST processing (OSIC) for low SNR as can be seen in Figure 8. Additionally, trellis encoding (for 4-QAM) and Viterbi receiver were also superficially tested in this MIMO OFDM V-BLAST scheme. Both schemes showed a slightly better performance than Hamming $(n, k)$ coding/encoding which would not justify the increase of complexity in the system.

\section{MIMO-OFDM SYSTEM COMPLEXITY}

The implementation complexity of the MIMO system represents a substantial increase over existing devices. There are two primary areas of increased complexity associated with the MIMO system: RF and baseband processing. An assessment of FPGA implementation shows that a $2 \times 2$ implementation is roughly three times the baseband complexity of current devices. A $4 \times 4$ implementation is about eight times the baseband complexity of current devices. Given the continuous increase in transistor density, the baseband processing is not a significant cost driver for next-generation technology. While additional RF receive and transmit chains are required to support MIMO, some parts of the chains, such as the local oscillator and clock generation circuitry, can be shared. In addition, the transmit power requirement per power amplifier decreases directly in proportion to the number of antennas employed. Improved support for sleep and idle modes in the MAC will permit highly efficient power utilization for battery-powered devices, achieving charge cycles equivalent to or better than those cell phones achieve today.

To further study the impact of the multiple antennas, Fig. 7 shows the gain in MSE for a MIMO system over the SISO system as function of the rms delay spread for an average SNR of $20 \mathrm{~dB}$. Clearly the highest gain is achieved at low delay spreads. For high rms delay spreads the improvement goes towards the improvement found for AWGN, i.e. $10 \log 10(\mathrm{Nr}) \mathrm{dB}$. The decrease in improvement as function of delay spread is caused by the increasing frequency diversity. When the frequency diversity is high, the addition of TX or RX diversity does not gain significantly compared to the gain in case of AWGN. The results also show that the gain of the MIMO configurations is more than $10 \log 10(\mathrm{Nr}) \mathrm{dB}$ higher than the corresponding case with only TX diversity, showing the influence of the mentioned MRC like performance. The impact of the proposed frequency synchronization approach on the overall system performance is illustrated by means of an example in Fig. 6. It shows the raw biterror- rate (BER) (without 
coding) for $1 \times 1,2 \times 2$ and $4 \times 4$ systems, respectively, applying VBLAST [17] as MIMO detection algorithm for both perfect synchronization and for the implementation of the above described synchronization.

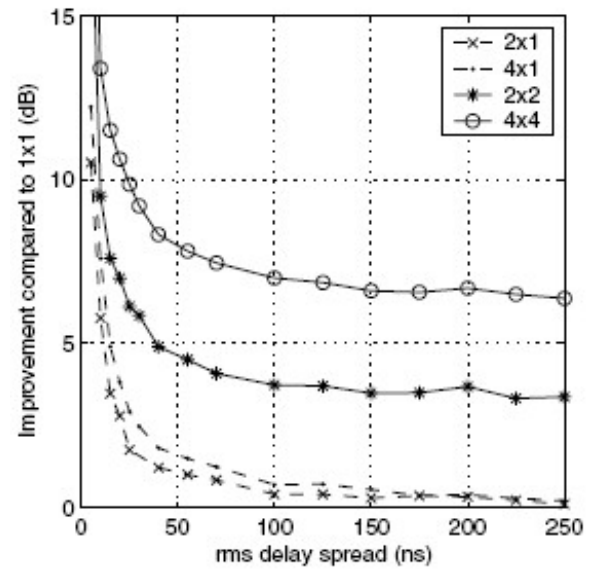

FIGURE 7. Improvement in MSE for different configurations compared to $1 \times 1$.

The packet length is $16 \mu$ s, i.e. 4 MIMO OFDM symbols, independent of the MIMO configuration. For the simulations, uncorrelated multipath channels with a rms delay spread of $50 \mathrm{~ns}$ are applied. To show only the influence of the frequency synchronization, the channel estimation is assumed perfect.

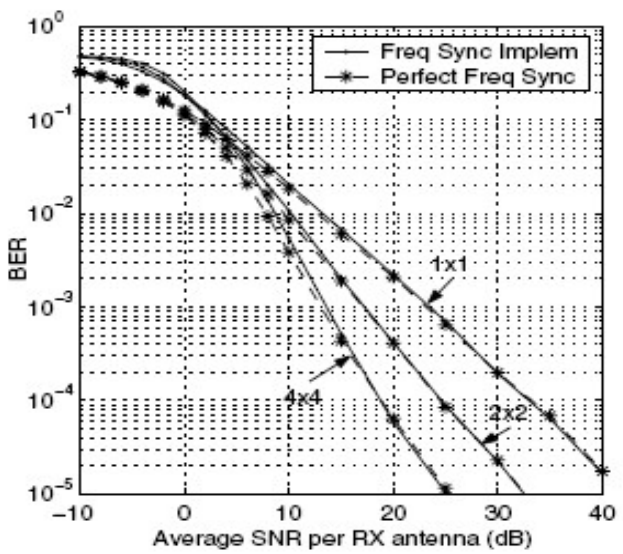

FIGURE 8. Performance of VBLAST algorithm applying perfect $(-)$ and implemented synchronization $(-)$ for a $1 \times 1,2 \times 2$ and $4 \times 4$ configuration.

It is clear from the results in Fig. 8 that the implementation of the frequency synchronization causes some degradation in performance at low SNR, which can be explained by the poor estimation of the frequency offset at these SNR values. In the BER range of interest, however, the degradation compared to perfect synchronization is very small.

\section{BUILDING FUTURE NETWORKS WITH MIMO AND OFDM}

It is important to note that the OFDM-MIMO evolution being suggested here for 3GPP and 3GPP2 is an evolution of existing 3G networks - not a new network. This means existing sites and network architecture could be re-used, allowing wireless operators to preserve their previous investments in existing networks like today's $3 \mathrm{G}$ networks. On the other hand, the use of OFDM-MIMO for WiMAX 802.16e, which is progressing very rapidly, would require a new network. 
The building of large-scale OFDM-MIMO networks will take several years and it will not be without challenges. For instance, at the cell site, the use of cross pole antennas will allow service providers to avoid having to install additional large antennas, which can cause installation and zoning problems. However, service providers and equipment manufacturers will need cabling from the antennas to the tower bases. Devices will also be impacted, requiring extra built-in antennas and more stringent component tolerances. Fortunately, significant work is in progress to address these issues.

OFDM has some key advantages over the common wireless access technology known as CDMA, which is used in many of today's $3 \mathrm{G}$ cellular networks. To begin with, it is more robust, which means that it provides better performance in cluttered areas with many reflections (multipath). It also allows for simpler receivers. Perhaps most important, OFDM is more amenable to MIMO technologies. A trial conducted in Nortel's Wireless Technology Lab in early 2005 offers an example of this synergistic nature. During the trial, a mobile user had the ability to view two live streaming videos simultaneously while downloading a $264 \mathrm{MB}$ file at $37 \mathrm{Mb} / \mathrm{s}$ over a standard $5 \mathrm{MHz}$ PCS band. Using OFDM-MIMO, the download was achieved in less than a minute compared to the 90 minutes that would be required with today's networks. This is roughly 10 times the $3.6 \mathrm{Mbps}$ enabled by the first generation of HSDPA devices.

OFDM is a logical next step in broadband radio evolution. It is already being applied in IEEE standards like IEEE 802.11 and 802.16, also referred to as Wi-Fi and WiMAX, respectively. Standards groups are currently working to standardize OFDM-MIMO as it relates to Wi-Fi and WiMAX. At this time, OFDM-MIMO is not part of the formal evolution path for existing cellular systems based on the 3GPP (UMTS, HSDPA) and 3GPP2 (CDMA 1X, EV-DO) standards; however, standards groups are working to understand its role in providing wireless broadband.

\section{CONCLUSION}

There is considerable evidence that the appetite for wireless broadband is only just beginning and that wireless broadband will become the largest growth area within the wireless industry. The starting point for this growth is increasing consumer reliance on laptop computers. Today, more laptops are sold than desktop PCs. As this trend continues, so will users' demand for the same type of broadband service no matter where they are - at home, at the office, or on the road.

WiFi and existing high-speed cellular networks being deployed today meet some of these needs, but OFDMMIMO, used by WiMAX 802.16 e or beyond 3G, is the technology needed to allow for economic and scalable wireless broadband. The scalability furnished by these technologies becomes especially important once MP3 players, PDAs, portable games devices and other handheld devices are equipped to provide wireless broadband, giving users a broadband connection wherever they go.

Going back to our earlier analogy, if the wireless industry fast-forwards the acceptance of MIMO and OFDM technologies into the refinement of our highways, we will be able to put the latest, greatest automobiles-applications--on those highways and allow people to get places faster and more efficiently than ever before. In this way, MIMO and OFDM are key technologies for enabling the wireless industry to deliver on the vast potential and promise of wireless broadband.

\section{REFERENCES}

1. C. V. Seshaiah, S. Nagarani "A Survey on Space-Time Turbo Codes" IJCSIS, Vol. 7 No. 3, March 2010, 171 177, ISSN 1947 5500J. Clerk Maxwell, A Treatise on Electricity and Magnetism, 3rd ed., vol. 2. Oxford: Clarendon, 1892, pp.68-73.

2. L. Hanzo, S. X. Ng, T. Keller, and W. T. Webb, Quadrature AmplitudeModulation: From Basics to Adaptive Trellis- Coded, Turbo-Equalised and Space-Time Coded OFDM, CDMA and MC-CDMA Systems,3rd ed. Piscataway, NJ: IEEE Press/Wiley,2004.

3. R. V. Nee and R. Prasad, OFDM for Wireless Multimedia Communications. London, U.K.: Artech House, 2000.

4. J. A. C. Bingham, BMulticarrier modulation for data transmission: An idea whose time has come, [ IEEE Commun. Mag., vol. 28, no. 5, pp. 5-14, May 2011.

5. P. Coulton and D. Carline, "An SDR inspired design for the FPGA implementation of 802.11a baseband system," Proceeding. of IEEE International Symposium on Consumer Electronics, pp. 470-475, Sep 2012. 
6. C. Dick and F. Harris, "FPGA implementation of an OFDM PHY," Conference Record of the Thirty Seventh Asilomar Conference on Signals, Systems and Computers, vol. 1, pp. 905-909, Nov 2012.

7. G. Jongren and M. Skoglund and B. Ottersten, "Combining beam forming and orthogonal space-time block coding," IEEE Transactions on Information Theory, vol. 48, no. 3, pp.611.627, Mar 2012.

8. H. Bolcskei and D. Gesbert and A. J. Paulraj "On the capacity of OFDM-Based Spatial Multiplexing systems," IEEE Trans. Commun., vol. 50, no. 2, pp. 225-234, Feb 2012.

9. H. Liu and U. Tureli, "A high efficiency carrier estimator for OFDM communications", IEEE Communication Letters, vol. 2, no. 4, pp. 104-106, April 1998.

10. J. van de Beek, M. Sandell and P.O. Borjesson," ML estimation of time and frequency offset in OFDM systems", IEEE Trans. on Signal Processing, vol. 45, no. 7, pp. 1800-1805, July 1997. 